\title{
openheart Lauric acid-rich medium-chain triglycerides can substitute for other oils in cooking applications and may have limited pathogenicity
}

\author{
Mark F McCarty, James J DiNicolantonio
}

To cite: McCarty MF, DiNicolantonio JJ. Lauric acid-rich medium-chain triglycerides can substitute for other oils in cooking applications and may have limited pathogenicity. Open Heart 2016;3:e000467. doi:10.1136/openhrt-2016000467

Received 3 May 2016 Revised 9 June 2016 Accepted 2 July 2016

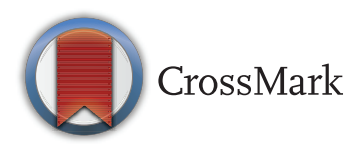

Catalytic Longevity, Encinitas, California, USA

Correspondence to Dr Mark F McCarty; markfmccarty@gmail.com

\section{ABSTRACT}

Recently, medium-chain triglycerides (MCTs) containing a large fraction of lauric acid (LA) (C12) about 30\% - have been introduced commercially for use in salad oils and in cooking applications. As compared to the long-chain fatty acids found in other cooking oils, the medium-chain fats in MCTs are far less likely to be stored in adipose tissue, do not give rise to 'ectopic fat' metabolites that promote insulin resistance and inflammation, and may be less likely to activate macrophages. When ingested, medium-chain fatty acids are rapidly oxidised in hepatic mitochondria; the resulting glut of acetyl-coenzyme A drives ketone body production and also provokes a thermogenic response. Hence, studies in animals and humans indicate that MCT ingestion is less obesogenic than comparable intakes of longer chain oils. Although LA tends to raise serum cholesterol, it has a more substantial impact on high density lipoprotein (HDL) than low density lipoprotein (LDL) in this regard, such that the ratio of total cholesterol to $\mathrm{HDL}$ cholesterol decreases. LA constitutes about $50 \%$ of the fatty acid content of coconut oil; south Asian and Oceanic societies which use coconut oil as their primary source of dietary fat tend to be at low cardiovascular risk. Since ketone bodies can exert neuroprotective effects, the moderate ketosis induced by regular MCT ingestion may have neuroprotective potential. As compared to traditional MCTs featuring C6-C10, laurate-rich MCTs are more feasible for use in moderate-temperature frying and tend to produce a lower but more sustained pattern of blood ketone elevation owing to the more gradual hepatic oxidation of ingested laurate.

\section{TRIGLYCERIDES SYNTHESISED FROM COCONUT OIL}

Standard medium-chain triglycerides (MCTs) are produced by hydrolysing coconut oil and esterifying the fatty acids shorter than lauric acid (LA) (C12) with glycerol; the resulting triglycerides are rich primarily in caprylic (C8) and capric (C10) acids. The exclusion of LA reflects the fact that this fatty acid has high commercial value as a precursor for antibacterial pharmaceuticals (eg, monolaurin) and other worthwhile compounds. Coconut oil is one of the richest available sources of LA-constituting about half of its total fatty acid content-and so is used to produce LA; the shorter chain fats are hence by-products of this process and then are used for production of MCTs. As contrasted with coconut oil, standard MCTs are consistently fluid at room temperature; their utility for cooking applications, however, is limited by their low smoke point, which makes them unsuitable for use in frying.

Recently, however, manufacturers have started to produce a novel type of MCT that contains a high fraction of LA-typically $30 \%$. A tablespoon of this MCT-containing $14 \mathrm{~g}$ of fat-is said to contain $12 \mathrm{~g}$ of medium-chain fatty acids (lauric $4.45 \mathrm{~g}$, caprylic $3.35 \mathrm{~g}$, capric $4.00 \mathrm{~g}$ ) and $1 \mathrm{~g}$ of unsaturates (presumably largely oleic acid). Hence, the content of longer chain saturated fatty acids is extremely low and of questionable physiological significance.

\section{METABOLIC FATES OF MEDIUM-CHAIN TRIGLYCERIDES}

The fatty acids featured in MCTs are characterised by a limited potential for storage as triglycerides. This reflects the fact that they cannot be employed for de novo synthesis of diacylglycerol or phosphatidic acid. ${ }^{1} \quad{ }^{2}$ However, they can act as substrates, to a limited extent, for diacylglycerol acyltransferase; laurate is more active in this regard than the shorter chain fatty acids. ${ }^{12}$ This means that medium-chain fatty acids can participate in triglyceride synthesis when other longer chain fatty acids are present to generate diacylglycerol.

The half life of ingested medium-chain fatty acids (MCFAs) tends to be short not 
only because the capacity for their storage is less than that for longer chain fats but also because they can enter mitochondria efficiently without preliminary esterification to carnitine. ${ }^{3}$ Conversion of fatty acylcoenzyme As to fatty acyl-carnitines in the cytoplasm (via carnitine palmitoyltransferase-I-CPT-I) is a process tightly regulated in regard to metabolic need. When cellular glucose availability is ample, CPT-I is inhibited by malonyl-coenzyme A; insulin activity also inhibits this enzyme. ${ }^{4}$ Ketosis only develops when the liver is glycogen depleted and insulin levels are low, such that CPT-I activity is disinhibited. ${ }^{5}$ Under these circumstances, fatty acids in the portal circulation have rapid access to the inner matrix of mitochondria in hepatocytes; their subsequent oxidative degradation gives rise to a glut of acetyl-coenzyme A, some of which will be converted to ketone bodies that enter the circulation. A bolus dose of MCTs likewise can give rise to ketone production in hepatocytes-even in the context of ample glycogen availability-because medium-chain fatty acids can stream into hepatic mitochondria efficiently, where they are rapidly converted either to ketone bodies or to $\mathrm{CO}_{2} .{ }^{3}$ (Insulin/glucagon balance, however, can still partially regulate ketone production as it can influence the activity and expression of the rate-limiting enzyme for ketone body production, HMG-coenzyme A synthetase ${ }^{6}$ hence, a somewhat higher proportion of medium-chain fatty acids may be converted to ketone bodies during fasting metabolism).

The high ketogenicity of C8 and C10 further reflects the fact that, as they are poorly incorporated into chylomicrons but are relatively soluble, they tend to enter the portal circulation directly after absorption (as opposed to the lymphatics) and hence have rapid access to the liver. Rodent studies show that LA has a higher propensity to be absorbed via the lymphatics (presumably reflecting its greater capacity for incorporation into triglycerides), and so its access to the liver is delayed. ${ }^{78}$ Logically, this delay should imply that the rise of ketone bodies after laurate ingestion is delayed and that a somewhat lower proportion of this fatty acid (as compared to C8 or C10) is ultimately converted to ketones; the latter can be deduced from the fact that a sudden glut of hepatic mitochondrial acetyl-coenzyme A is more productive of ketones than a small sustained rise. Data of Veech cited by Newport do indeed indicate that, whereas administration of a bolus of standard MCT oil produces a large rise in plasma ketones that returns to baseline levels within about 3 hours, ingestion of intact coconut oil (in which laurate is the chief MCFA) leads to a delayed and less prominent rise in ketone bodies. ${ }^{9}$

\section{HEALTH ADVANTAGES OF MEDIUM-CHAIN FATTY ACIDS}

The fact that MCFAs are less efficiently stored than other fatty acids and are highly prone to oxidative metabolism once ingested, implies that they have a short half life in the body and are unlikely to promote obesity via direct storage in adipocytes. ${ }^{10}$ Moreover, bolus ingestion of MCTs tends to trigger thermogenesis, presumably reflecting the fact that a glut of acetyl-coenzyme A production in mitochondria tends to trigger protective uncoupling mechanisms. ${ }^{11-13}$ Studies in rodents and humans indicate that, when diets are fed containing comparable amounts of MCTs or longer chain fats, the MCT diets are less obesogenic. ${ }^{14}$ Hence, it has been proposed that MCTs should be used as an oil source by people who are attempting to control their weights. ${ }^{13}$

The adverse impact of excessive fatty acid exposure on health-especially long-chain saturated fatty acids-is attributable not only to modulation of serum lipid profile, or promotion of obesity but also to the production of 'ectopic fat' metabolites within tissues that interfere with insulin signalling and promote inflammation. ${ }^{15-19}$ Ceramide and diacylglycerol appear to be prominent in this regard. The production of these metabolites tends to be greater in obese people with an insulin-resistant fat depot, especially when they consume diets rich in fat and carbohydrates; indeed, these metabolites are suspected to mediate many of the adverse effects of metabolic syndrome. ${ }^{20-23}$ It is notable that MCFAs are incapable of giving rise to such metabolites. ${ }^{12}$ A number of studies in rats or humans have found that, as contrasted with longchain fatty acids, diets featuring MCFAs are less likely to induce insulin resistance ${ }^{24-27}$ - albeit a few studies conclude otherwise. $^{28}$

Longer chain saturated fatty acids have the ability to upregulate activation of macrophages/microglia via promotion of toll-like receptor signalling and by supporting ceramide synthesis. ${ }^{29-32}$ Conceivably, this helps to rationalise the many epidemiological studies associating metabolic syndrome and long-chain saturate-rich diets with increased risk for neurodegenerative disorders and atherogenesis. ${ }^{33} 34$ Although MCFAs cannot give rise to palmitate and have not promoted macrophage activation in some studies, other researchers report that, especially under low-serum conditions, LA can activate macrophages by promoting signalling via certain toll-like receptors-TLR2 heterodimers and TLR4 homodimers. ${ }^{29} 35$ Also, macrophages can express a receptor for MCFAs, GPR84, which can exert a pro-inflammatory effect. ${ }^{36}$ Whether these in vitro findings are pertinent to orally administered MCFAs is unclear; several rodent studies find that orally administered MCTs to rodents exert anti-inflammatory effects in certain contexts. ${ }^{37-39}$

With respect to lipoprotein metabolism, diets rich in LA tend to raise low density lipoprotein (LDL) levels, but they have a greater proportional effect on high density lipoprotein (HDL) levels, such that the total cholesterol/HDL cholesterol level declines; in fact, laurate is reported to have a greater depressive effect on this prognostically significant ratio than other fats. ${ }^{40} \mathrm{~A}$ meta-analysis of clinical feeding trials found that, whereas replacing $1 \%$ of dietary energy as carbohydrate with LA raises apoB non-significantly by $5.6 \mathrm{mg} / \mathrm{L}$, it raises apoA-1 by a significant $13.8 \mathrm{mg} / \mathrm{L} .{ }^{40} \mathrm{It}$ is notable 
that, in South Seas cultures in which coconuts (and hence LA) are the predominant dietary fat source, cardiovascular disease tends to be relatively rare. ${ }^{41-44}$

Hence, as a dietary oil, MCTs or laurate-rich MCTs can be recommended for the following reasons: they are unlikely to exacerbate obesity; they do not give rise to ectopic fat metabolites that are key mediators of the pathogenicity of metabolic syndrome; and their impact on serum lipid profile appears to be relatively benign, despite an increase in total cholesterol.

\section{NEUROPROTECTIVE POTENTIAL OF KETONE BODIES}

Moreover, ketone bodies have neuroprotective potential, ${ }^{45-49}$ and a diet rich in MCTs represents a convenient means to raise plasma ketone body levels without the undertaking the inconvenience and monotony of severe carbohydrate restriction. ${ }^{50}$ Although the rise in plasma ketones achieved with MCTs is much less dramatic than that which can be achieved during prolonged fasting or carbohydrate avoidance, there is reason to suspect that it may be sufficient to aid cognitive function in elderly patients with minimal cognitive dysfunction or early Alzheimer's disease (AD). ${ }^{9}$ Indeed, small studies in rodents, dogs and humans support this conclusion, and a MCT preparation has been approved as a 'medical food' for use in $\mathrm{AD} .^{52-60}$ Decreased neuronal usage of glucose in brain regions affected in $\mathrm{AD}$ is a key feature of presymptomatic $\mathrm{AD},{ }^{61-63}$ and it has been postulated that, by serving as an alternate source of biochemical energy for brain neurons, ketone bodies may alleviate a neuronal 'energy deficit' in $\mathrm{AD}$, thereby improving cognitive function. ${ }^{64}$ Ketone bodies also have the potential to aid production of acetylcholine and hence may address the cholinergic deficit in $\mathrm{AD} .{ }^{66}{ }^{67}$ Whether moderate ketosis achievable with MCTs might have an impact on the fundamental pathogenic process in $\mathrm{AD}$ and other prominent neurodegenerative conditions, perhaps delaying or slowing the progression of these syndromes, is not yet clear; in any case, symptomatic benefit in $\mathrm{AD}$-as seen with cholinesterase inhibitor drugs-appears likely with an adequate intake of MCTs. (Administration of so-called 'ketone esters', which can replicate the ketone levels seen during fasting, may be required to achieve the fullest neuroprotective benefits of ketone bodies). ${ }^{68} 69$

When attempting to use dietary MCFAs to promote ketogenesis for health reasons, laurate-rich MCTs may have the advantage that they combine the rapid-acting C8 and C10 with the more delayed-acting LA; hence, they might be expected to produce a more sustained and moderate rise in plasma ketone bodies, as opposed to the large episodic rises and falls which ingestion of standard MCTs would tend to produce.

\section{USING LAURATE-RICH MCTS: PRACTICAL CONSIDERATIONS}

The main drawback with standard MCTs as a cooking oil is the fact that it is not considered safe or appropriate for use in frying owing to a low smoke point. ('Smoke point' refers to the temperature at which triglycerides degrade, producing soot and off-flavours.) Laurate-rich MCTs have the advantage that they can be expected to have a somewhat higher smoke point and hence can be employed in home pan frying and sauteeing (albeit not deep frying). Hence, laurate-rich MCTs appear to be appropriate for use in most home cooking applications.

When taken as a bolus-for producing ketones, for example- too high a dose of MCTs tends to produce diarrhoea and gastrointestinal (GI) upset. It seems logical to expect that this effect will be less notable with laurate-rich MCTs, which are closer in structure to coconut oil. Anecdotally, there appear to be few if any reports of GI intolerance when laurate-rich MCTs are used as a cooking oil.

Perhaps the chief hindrance to the widespread applications of laurate-rich MCTs is cost; current retail price for a litre is about US\$32. Presumably, the fast food industry and mass food manufacturers would not be interested in such a pricey oil; but motivated consumers who have a reasonable income could choose to afford it for home cooking applications.

A whole-food, fully plant-based diet, with no added oils, accompanied by standard pharmacotherapy, has been found to have remarkable efficacy for preventing further vascular events in patients with advanced coronary disease. ${ }^{70-72}$ Getting patients to abstain from all animal products is a difficult enough proposition, and the additional proscription of added oils makes it all the harder as fat has a major impact on flavour. The possibility that laurate-rich MCTs might be used safely with such regimens is worthy of consideration.

Contributors MFM wrote the original draft, and JJD contributed revisions and additional ideas.

Competing interests None declared.

Provenance and peer review Not commissioned; externally peer reviewed.

Open Access This is an Open Access article distributed in accordance with the Creative Commons Attribution Non Commercial (CC BY-NC 4.0) license, which permits others to distribute, remix, adapt, build upon this work noncommercially, and license their derivative works on different terms, provided the original work is properly cited and the use is non-commercial. See: http:// creativecommons.org/licenses/by-nc/4.0/

\section{REFERENCES}

1. Mayorek N, Bar-Tana J. Medium chain fatty acids as specific substrates for diglyceride acyltransferase in cultured hepatocytes. J Biol Chem 1983;258:6789-92.

2. Brandes R, Mayorek N, Berry E, et al. The specificity of triacylglycerol synthesis for medium-chain fatty acids in rat and human adipose preparations. Biochim Biophys Acta 1985;836:63-6.

3. Hoppel CL. Carnitine and carnitine palmitoyltransferase in fatty acid oxidation and ketosis. Fed Proc 1982;41:2853-7.

4. McGarry JD, Brown NF. The mitochondrial carnitine palmitoyltransferase system. From concept to molecular analysis Eur J Biochem 1997;244:1-14.

5. Foster DW, McGarry JD. The regulation of ketogenesis. Ciba Found Symp 1982;87:120-31.

6. Hegardt FG. Transcriptional regulation of mitochondrial HMG-coA synthase in the control of ketogenesis. Biochimie 1998;80:803-6. 
7. Sigalet DL, Winkelaar GB, Smith LJ. Determination of the route of medium-chain and long-chain fatty acid absorption by direct measurement in the rat. JPEN J Parenter Enteral Nutr 1997;21:275-8

8. Sigalet DL, Martin G. Lymphatic absorption of glucose and fatty acids as determined by direct measurement. J Pediatr Surg 1999;34:39-43.

9. Newport MT. Alzheimer's disease-what if there were a cure? 2nd edn. Laguna Beach: Basic Health Publications, 2013.

10. Bach AC, Ingenbleek $Y$, Frey A. The usefulness of dietary medium-chain triglycerides in body weight control: fact or fancy? $J$ Lipid Res 1996;37:708-26.

11. Hill JO, Peters JC, Yang D, et al. Thermogenesis in humans during overfeeding with medium-chain triglycerides. Metabolism 1989;38:641-8.

12. Ogawa A, Nosaka N, Kasai M, et al. Dietary medium- and long-chain triacylglycerols accelerate diet-induced thermogenesis in humans. J Oleo Sci 2007;56:283-7.

13. Papamandjaris AA, MacDougall DE, Jones PJ. Medium chain fatty acid metabolism and energy expenditure: obesity treatment implications. Life Sci 1998;62:1203-15.

14. Mumme K, Stonehouse W. Effects of medium-chain triglycerides on weight loss and body composition: a meta-analysis of randomized controlled trials. J Acad Nutr Diet 2015;115:249-63.

15. Szendroedi J, Yoshimura T, Phielix E, et al. Role of diacylglycerol activation of PKCtheta in lipid-induced muscle insulin resistance in humans. Proc Natl Acad Sci USA 2014;111:9597-602.

16. Jornayvaz FR, Shulman Gl. Diacylglycerol activation of protein kinase Cepsilon and hepatic insulin resistance. Cell Metab 2012;15:574-84.

17. Gao D, Pararasa C, Dunston CR, et al. Palmitate promotes monocyte atherogenicity via de novo ceramide synthesis. Free Radic Biol Med 2012;53:796-806.

18. Mugabo Y, Mukaneza Y, Renier G. Palmitate induces C-reactive protein expression in human aortic endothelial cells. Relevance to fatty acid-induced endothelial dysfunction. Metabolism 2011;60:640-8.

19. Xiao-Yun $X$, Zhuo-Xiong $C$, Min-Xiang $L$, et al. Ceramide mediates inhibition of the AKT/eNOS signaling pathway by palmitate in human vascular endothelial cells. Med Sci Monit 2009;15:BR254-61.

20. Shimabukuro M, Kozuka C, Taira S, et al. Ectopic fat deposition and global cardiometabolic risk: new paradigm in cardiovascular medicine. J Med Invest 2013;60:1-14

21. Taira $S$, Shimabukuro $M$, Higa $M$, et al. Lipid deposition in various sites of the skeletal muscles and liver exhibits a positive correlation with visceral fat accumulation in middle-aged Japanese men with metabolic syndrome. Intern Med 2013;52:1561-71.

22. Guebre-Egziabher F, Alix PM, Koppe L, et al. Ectopic lipid accumulation: A potential cause for metabolic disturbances and a contributor to the alteration of kidney function. Biochimie 2013;95:1971-9.

23. Smith U. Abdominal obesity: a marker of ectopic fat accumulation J Clin Invest 2015;125:1790-2.

24. Wilson DE, Chan IF, Stevenson KB, et al. Eucaloric substitution of medium chain triglycerides for dietary long chain fatty acids in acquired total lipodystrophy: effects on hyperlipoproteinemia and endogenous insulin resistance. J Clin Endocrinol Metab 1983;57:517-23.

25. St-Onge MP, Bosarge A, Goree LL, et al. Medium chain triglyceride oil consumption as part of a weight loss diet does not lead to an adverse metabolic profile when compared to olive oil. J Am Coll Nutr 2008;27:547-52.

26. Wein S, Wolffram S, Schrezenmeir J, et al. Medium-chain fatty acids ameliorate insulin resistance caused by high-fat diets in rats. Diabetes Metab Res Rev 2009;25:185-94.

27. Nagao K, Yanagita T. Medium-chain fatty acids: functional lipids for the prevention and treatment of the metabolic syndrome. Pharmacol Res 2010;61:208-12.

28. De Vogel-van den Bosch J, van den Berg SA, et al. High-fat diets rich in medium- versus long-chain fatty acids induce distinct patterns of tissue specific insulin resistance. J Nutr Biochem 2011;22:366-71.

29. Huang S, Rutkowsky JM, Snodgrass RG, et al. Saturated fatty acids activate TLR-mediated proinflammatory signaling pathways. J Lipid Res 2012;53:2002-13.

30. Little JP, Madeira JM, Klegeris A. The saturated fatty acid palmitate induces human monocytic cell toxicity toward neuronal cells: exploring a possible link between obesity-related metabolic impairments and neuroinflammation. J Alzheimers Dis 2012;30 (Suppl 2):S179-83.

31. Tracy LM, Bergqvist F, Ivanova EV, et al. Exposure to the saturated free fatty acid palmitate alters BV-2 microglia inflammatory response. J Mol Neurosci 2013;51:805-12.
32. Håversen L, Danielsson KN, Fogelstrand L, et al. Induction of proinflammatory cytokines by long-chain saturated fatty acids in human macrophages. Atherosclerosis 2009;202:382-93.

33. Morris MC, Tangney CC. Dietary fat composition and dementia risk. Neurobiol Aging 2014;35(Suppl 2):S59-64.

34. Barnard ND, Bunner AE, Agarwal U. Saturated and trans fats and dementia: a systematic review. Neurobiol Aging 2014;35(Suppl 2): S65-73.

35. Lee JY, Zhao L, Youn HS, et al. Saturated fatty acid activates but polyunsaturated fatty acid inhibits Toll-like receptor 2 dimerized with toll-like receptor 6 or 1. J Biol Chem 2004;279:16971-9.

36. Suzuki M, Takaishi S, Nagasaki M, et al. Medium-chain fatty acid-sensing receptor, GPR84, is a proinflammatory receptor. J Biol Chem 2013;288:10684-91.

37. Kono $\mathrm{H}$, Fujii $\mathrm{H}$, Asakawa $\mathrm{M}$, et al. Protective effects of medium-chain triglycerides on the liver and gut in rats administered endotoxin. Ann Surg 2003;237:246-55.

38. Kono $\mathrm{H}$, Enomoto $\mathrm{N}$, Connor HD, et al. Medium-chain triglycerides inhibit free radical formation and TNF-alpha production in rats given enteral ethanol. Am J Physiol Gastrointest Liver Physiol 2000;278: G467-76.

39. Carlson SJ, Nandivada P, Chang MI, et al. The addition of medium-chain triglycerides to a purified fish oil-based diet alters inflammatory profiles in mice. Metabolism 2015;64:274-82.

40. Mensink RP, Zock PL, Kester AD, et al. Effects of dietary fatty acids and carbohydrates on the ratio of serum total to HDL cholesterol and on serum lipids and apolipoproteins: a meta-analysis of 60 controlled trials. Am J Clin Nutr 2003;77:1146-55.

41. Prior IA, Davidson F, Salmond CE, et al. Cholesterol, coconuts, and diet on Polynesian atolls: a natural experiment: the Pukapuka and Tokelau island studies. Am J Clin Nutr 1981;34:1552-61.

42. Lindeberg S, Lundh B. Apparent absence of stroke and ischaemic heart disease in a traditional Melanesian island: a clinical study in Kitava. J Intern Med 1993;233:269-75.

43. Lipoeto NI, Agus Z, Oenzil F, et al. Dietary intake and the risk of coronary heart disease among the coconut-consuming Minangkabau in West Sumatra, Indonesia. Asia Pac J Clin Nutr 2004;13:377-84.

44. Kumar PD. The role of coconut and coconut oil in coronary heart disease in Kerala, south India. Trop Doct 1997;27:215-17.

45. Cahill GF Jr, Veech RL. Ketoacids? Good medicine? Trans Am Clin Climatol Assoc 2003;114:149-61.

46. Gasior M, Rogawski MA, Hartman AL. Neuroprotective and disease-modifying effects of the ketogenic diet. Behav Pharmacol 2006;17:431-9.

47. Maalouf M, Rho JM, Mattson MP. The neuroprotective properties of calorie restriction, the ketogenic diet, and ketone bodies. Brain Res Rev 2009:59:293-315.

48. Offermanns S, Schwaninger M. Nutritional or pharmacological activation of $\mathrm{HCA}(2)$ ameliorates neuroinflammation. Trends $\mathrm{Mol}$ Med 2015;21:245-55.

49. Paoli A, Bianco A, Damiani E, et al. Ketogenic diet in neuromuscular and neurodegenerative diseases. Biomed Res Int 2014;2014:474296.

50. Freund G, Weinsier RL. Standardized ketosis in man following medium chain triglyceride ingestion. Metabolism 1966;15:980-91.

51. Courchesne-Loyer A, Fortier M, Tremblay-Mercier J, et al. Stimulation of mild, sustained ketonemia by medium-chain triacylglycerols in healthy humans: estimated potential contribution to brain energy metabolism. Nutrition 2013;29:635-40.

52. Roman MW. Axona (Accera, Inc): a new medical food therapy for persons with Alzheimer's disease. Issues Ment Health Nurs 2010;31:435-6.

53. Kashiwaya $\mathrm{Y}$, Bergman $\mathrm{C}$, Lee $\mathrm{JH}$, et al. A ketone ester diet exhibits anxiolytic and cognition-sparing properties, and lessens amyloid and tau pathologies in a mouse model of Alzheimer's disease. Neurobiol Aging 2013;34:1530-9.

54. Mikkelsen $\mathrm{KH}$, Seifert $\mathrm{T}$, Secher $\mathrm{NH}$, et al. Systemic, cerebral and skeletal muscle ketone body and energy metabolism during acute hyper-D-beta-hydroxybutyratemia in post-absorptive healthy males $J$ Clin Endocrinol Metab 2015;100:636-43.

55. Reger MA, Henderson ST, Hale C, et al. Effects of beta-hydroxybutyrate on cognition in memory-impaired adults. Neurobiol Aging 2004;25:311-14.

56. Henderson ST, Vogel JL, Barr LJ, et al. Study of the ketogenic agent AC-1202 in mild to moderate Alzheimer's disease: a randomized double-blind, placebo-controlled, multicenter trial. Nutr Metab (Lond) 2009;6:31

57. Pan Y, Larson B, Araujo JA, et al. Dietary supplementation with medium-chain TAG has long-lasting cognition-enhancing effects in aged dogs. Br J Nutr 2010;103:1746-54. 
58. Maynard SD, Gelblum J. Retrospective case studies of the efficacy of caprylic triglyceride in mild-to-moderate Alzheimer's disease. Neuropsychiatr Dis Treat 2013;9:1629-35.

59. Farah BA. Effects of caprylic triglyceride on cognitive performance and cerebral glucose metabolism in mild Alzheimer's disease: a single-case observation. Front Aging Neurosci 2014;6:133.

60. Newport MT, Vanltallie TB, Kashiwaya Y, et al. A new way to produce hyperketonemia: use of ketone ester in a case of Alzheimer's disease. Alzheimers Dement 2015;11:99-103.

61. Hoyer S. Abnormalities of glucose metabolism in Alzheimer's disease. Ann N Y Acad Sci 1991;640:53-8.

62. Simpson IA, Chundu KR, Davies-Hill T, et al. Decreased concentrations of GLUT1 and GLUT3 glucose transporters in the brains of patients with Alzheimer's disease. Ann Neurol 1994;35:546-51.

63. Liu Y, Liu F, lqbal K, et al. Decreased glucose transporters correlate to abnormal hyperphosphorylation of tau in Alzheimer disease. FEBS Lett 2008;582:359-64.

64. Henderson ST. Ketone bodies as a therapeutic for Alzheimer's disease. Neurotherapeutics 2008;5:470-80.

65. Costantini LC, Barr LJ, Vogel JL, et al. Hypometabolism as a therapeutic target in Alzheimer's disease. BMC Neurosci 2008;9 (Suppl 2):S16.
66. Sterling GH, McCafferty MR, O'Neill JJ. Beta-hydroxybutyrate as a precursor to the acetyl moiety of acetylcholine. J Neurochem 1981;37:1250-9.

67. Tomaszewicz M, Bielarczyk $\mathrm{H}$, Jankowska $\mathrm{A}$, et al. Pathways of beta-hydroxybutyrate contribution to metabolism of acetyl-coA and acetylcholine in rat brain nerve terminals. Folia Neuropathol 1997;35:244-6.

68. Veech RL. Ketone ester effects on metabolism and transcription. J Lipid Res 2014;55:2004-6.

69. Clarke K, Tchabanenko K, Pawlosky R, et al. Oral 28-day and developmental toxicity studies of (R)-3-hydroxybutyl (R)-3-hydroxybutyrate. Regul Toxicol Pharmacol 2012;63:196-208.

70. Esselstyn CB Jr., Ellis SG, Medendorp SV, et al. A strategy to arrest and reverse coronary artery disease: a 5-year longitudinal study of a single physician's practice. J Fam Pract 1995;41:560-8.

71. Esselstyn CB Jr. Updating a 12-year experience with arrest and reversal therapy for coronary heart disease (an overdue requiem for palliative cardiology). Am J Cardiol 1999;84:339-41, A8.

72. Esselstyn CB Jr, Gendy G, Doyle J, et al. A way to reverse CAD? $J$ Fam Pract 2014;63:356-364b. 